Part of Journal of Research of the National Bureau of Standards, Volume 23, November 1939

\title{
PHOTOCHEMICAL DECOMPOSITION OF THE CYSTINE IN WOOL
}

\author{
By Henry A. Rutherford and Milton Harris ${ }^{1}$
}

\begin{abstract}
The work reported in the present paper is a continuation of earlier work on the photochemical decomposition of wool. The data confirm the hypothesis that the primary action of the radiation, which results in a change in the state of the sulfur and a corresponding lowering of the cystine content, is independent of the moisture in the system. The results indicate, however, that moisture plays an important role in the secondary process, namely that involving the evolution of hydrogen sulfide from the irradiated fibers. It is also shown that decomposition of the cystine resulting from thermal reactions under the conditions of these experiments is negligible.
\end{abstract}

\section{CONTENTS}

I. Introduction

II. Experimental procedure

1. Effect of light on the cystine in wool

2. Effect of temperature

3. Nature of primary and secondary reactions

III. Discussion_._.

IV. References

\section{INTRODUCTION}

In two earlier papers on the effect of light on wool [1, 2], ${ }^{2}$ it was shown that one of the chief points of attack is the disulfide group of the amino acid, cystine. The data suggested that the primary action of the radiation is an activation of the disulfide group, resulting in a change in state of the sulfur and a corresponding lowering of the cystine content. A secondary process, involving the evolution of hydrogen sulfide from the irradiated wool, then occurs. The rate of the primary reaction, that is, the decomposition of the cystine, appeared to be independent of the amount of water vapor in the system surrounding the fibers during irradiation. It was believed, however, that water might play an important part in the secondary reactions. The work reported in the present paper is a continuation of the earlier investigation.

1 Research Associates at the National Bureau of Standards, representing the Textile Foundation, Inc.

2 Figures in brackets indicate the literature references at the end of this paper. 


\section{EXPERIMENTAL PROCEDURE}

The apparatus, with the exception of a few minor changes described below, and technique of operation were the same as described in detail in an earlier paper [2]. The wool samples were irradiated in specially constructed tubes, in atmospheres of moist and dry nitrogen. The nitrogen, which was used as a carrier gas to remove the hydrogen sulfide formed during the irradiation, was first freed from oxygen by passing it through two towers of alkaline pyrogallol. The emerging gas was divided into two portions, one of which was moistened by passing it through distilled water, while the remainder was dried over anhydrous magnesium perchlorate and phosphorus pentoxide. From the drying and moistening towers, the gas passed through the exposure tubes and finally into an alkaline solution of bromine for absorption of hydrogen sulfide. The smooth elliptical aluminum reflector, used around the lamp and exposure tubes in the original apparatus, was replaced by a corrugated reflector in order to insure a more nearly uniform irradiation of the fibers. A stopcock was inserted between the tubes emerging from the drying and moistening towers so that both exposure tubes could be supplied with moist or with dry nitrogen at the same time.

Single-ply wool yarn of very low twist was used for the exposures. The yarn was prepared from raw wool which had been extracted with Stoddard solvent and washed with water. It was further purified by extraction in a Soxhlet apparatus with alcohol and ether, for 6 hours each, and finally washed with water at $50^{\circ} \mathrm{C}$.

\section{EFFECT OF LIGH'T ON THE CYSTINE IN WOOL}

Twenty 100-mg portions of the yarn were wound in adjoining layers on each of the two hexagonal glass frames. The wool on one frame was dried in an oven at $105^{\circ} \mathrm{C}$ for 3 hours, then rapidly transferred to the exposure tube, where it was further dried in a stream of dry nitrogen at $90^{\circ}$ to $100^{\circ} \mathrm{C}$ for 24 hours. The exposures were then started, dry nitrogen being passed over the dried sample and moist nitrogen over the other. During the exposures, the frames were rotated as described earlier [2]. After different time intervals, two of the 100-mg skeins, one from the top and one from the bottom of each frame, were removed from each of the exposure tubes and analyzed for cystine by the Sullivan method [3], using the hydrochloric-formic acid mixture for hydrolysis, as described by Miller and du Vigneaud [4]. The cystine contents of the samples after the exposures are shown in table 1 .

TABLE 1.-Cystine content of wool after irradiation in atmospheres of moist and of dry nitrogen

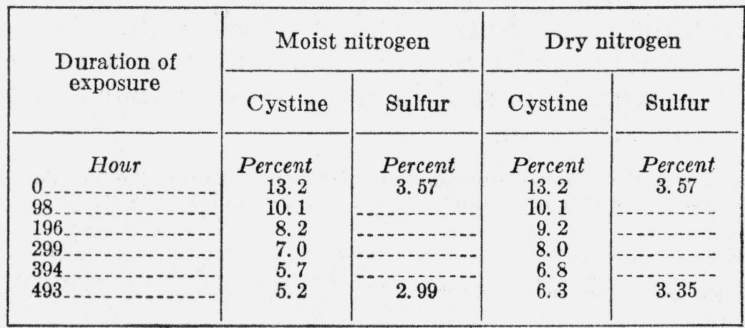




\section{EFFECT OF TEMPERATURE}

The temperature in the exposure tubes, as measured by a mercury thermometer (the bulb of which was covered with a thin layer of wool yarn), varied between $65^{\circ}$ and $70^{\circ} \mathrm{C}$. Since the temperature coefficient of most photochemical reactions is known to be small compared with that of most thermal reactions, it is hardly likely that the rate of decomposition of the cystine was appreciably affected by this small variation in temperature.

In order to determine whether some of the cystine was destroyed by thermal reactions alone during the long exposures, samples of wool were heated in the dark in atmospheres of moist and dry nitrogen at $60^{\circ}$ and $105^{\circ} \mathrm{C}$. An experiment was run at the higher temperature, since it was conceivable that the temperature on the surface of the fibers might be somewhat higher than that indicated by the thermometer. The wool yarn was placed in glass tubes in an oven; and nitrogen, dried or moistened by passing through towers of phosphorus pentoxide or water, was passed over the samples at the rate of about 3 liters per hour. The nitrogen was preheated to the desired temperature, after leaving the towers, by allowing it to pass through about $12 \mathrm{ft}$. of copper coil in the oven. After different time intervals, duplicate samples were removed for sulfur and cystine analyses, the results of which are shown in table 2.

TABLE 2.-Effect on the sulfur and cystine in wool of prolonged heating in the dark

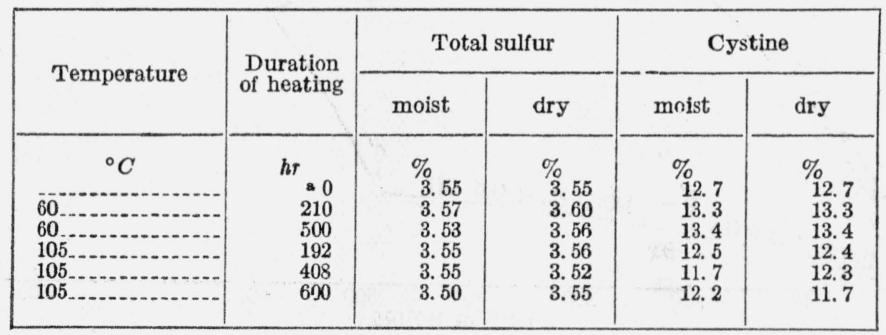

- Untreated wool.

\section{NATURE OF THE PRIMARY AND SECONDARY REACTIONS}

Samples of the wool yarn were wound on the glass frames and irradiated in atmospheres of moist and dry nitrogen for 200 hours. The lamp was then turned off, the temperature maintained at $70^{\circ} \mathrm{C}$, and moist nitrogen passed over both samples for about 300 hours. Finally, the lamp was again turned on and both samples were irradiated in atmospheres of moist nitrogen for an additional 300 hours. The rates at which sulfur, in the form of hydrogen sulfide, is evolved from the wool are shown in figure 1.

\section{DISCUSSION}

The data recorded in table 1 , showing the relative rates of decomposition of cystine during irradiation in atmospheres of moist and of dry nitrogen, confirm the earlier observation that the primary action of the radiation, which results in the decomposition of the cystine, is independent of the moisture in the system. The cystine contents 
of the samples irradiated in atmospheres of moist nitrogen are slightly lower; but in view of experimental difficulties, such as keeping the intensity of the light on both samples equal ${ }^{3}$ and constant, these small differences cannot be considered as significant at this time. Furthermore, the differences are extremely small when compared with the difference between the rates of evolution of hydrogen sulfide from wool irradiated in atmospheres of moist and of dry nitrogen.

The decomposition of the cystine under the conditions of these experiments appears to result from photochemical rather than thermal reactions. As was pointed out previously, the temperature in the

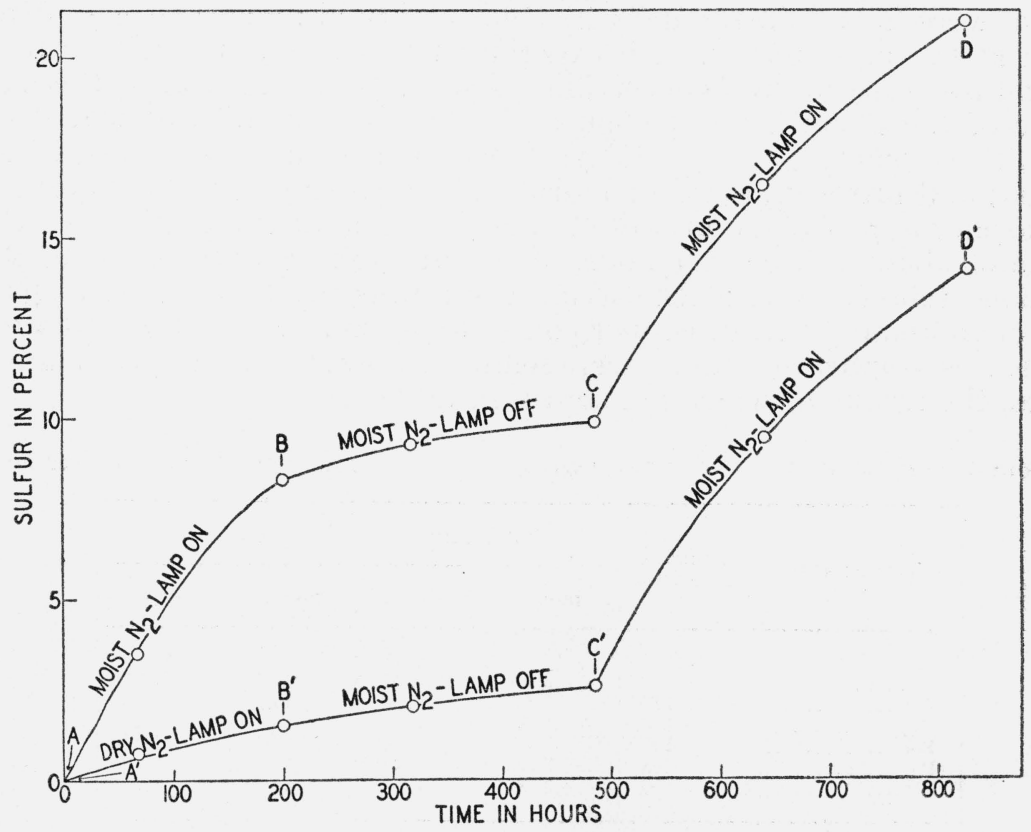

Figure 1.-Rate of evolution of hydrogen sulfide from wool.

The measurements were made (1) during irradiation in atmospheres of moist and dry nitrogen, (2) in the dark in atmospheres of moist nitrogen, the temperature being maintained at $70^{\circ} \mathrm{C}$, and (3) during irradiation in atmospheres of only moist nitrogen.

The ordinate values are in terms of the percentage of the total sulfur which was evolved as hydrogen sulfide during the treatment.

exposure tube varied between $65^{\circ}$ and $70^{\circ} \mathrm{C}$. However, the estimation of the exact temperature on the surface of the fiber would be very difficult, and for that reason an experiment in the absence of light was also run at $105^{\circ} \mathrm{C}$. The data in table 2 show that no significant changes in the sulfur or cystine contents of the wool occurred during the prolonged heating at $60^{\circ}$ and $105^{\circ} \mathrm{C}$.

Although the presence of moisture in the atmosphere surrounding the fibers during irradiation is not required for the primary reaction involving the decomposition of the cystine, the present work indicates that it does play an important role in the secondary process involving the evolution of the hydrogen sulfide from the irradiated fibers.

3 When samples were irradiated under the same conditions in both exposure tubes, hydrogen sulfide was evolved at the same rate. This was the basis for assuming equal intensity during subsequent experiments. 
Furthermore, it should be emphasized that the primary reaction is much more rapid than the secondary reaction and that the rate of evolution of hydrogen sulfide from the wool is determined by the rate of the secondary process. In addition, it appears that the secondary process is essentially a photochemical one, as indicated by the $B C D$ and $B^{\prime} C^{\prime} D^{\prime}$ portions of the curves in figure 1 . That a small amount of hydrogen sulfide is probably split from irradiated wool by thermal processes is indicated by the slopes of $B C$ and $B^{\prime} C^{\prime}$, but a comparison with those of the $C D$ and $C^{\prime} D^{\prime}$ portions shows that the amount is extremely small.

The authors can, at present, offer no complete explanation of the photochemical processes involved in the decomposition of the cystine in wool. The results to date suggest that the initial state of the photochemical decomposition is an absorption of the radiant energy resulting in the dissociation of the $\mathrm{R}-\mathrm{S}-\mathrm{S}-\mathrm{R}$ group, probably into $\mathrm{R}-\mathrm{S}^{+}$and $\mathrm{R}-\mathrm{S}^{-}$ions. This reaction does not appear to be catalyzed by moisture. A cleavage of this type for the photochemical decomposition of cystine itself has been postulated by Anslow and Foster [5], who showed that when radiant energy is absorbed by this substance, the linkage between the halves of the molecule is weakened, which results in the disruption of the molecule. A possible secondary reaction would then be the reaction of the products of the primary process with water to form $\mathrm{RSH}$ and $\mathrm{RSOH}$ compounds. The latter are unstable and generally decompose with the liberation of hydrogen sulfide.

\section{REFERENCES}

[1] A. L. Smith and M. Harris, J. Research NBS 1\%, 97 (1936) RP904.

[2] M. Harris and A. L. Smith, J. Research NBS 20, 563 (1938) RP1091.

[3] M. X. Sullivan, Public Health Reports No. 86 (1930).

[4] G. Miller and V. du Vigneaud, J. Biol. Chem. 118, 101 (1937).

[5] G. A. Anslow and M. L. Foster, J. Biol. Chem. 97, 37 (1932),

Washington, August 31, 1939. 